\title{
Accuracy of Lung Ultrasonography in Diagnosis of Community Acquired Pneumonia as Compared to Chest X-Ray in Pediatric Age Group
}

\author{
Abeer El-Sayed Metwally Mohamed, Omar Farouk Kamel, Mohamed Shaker Ghazy \\ Radiology Department, Faculty of Medicine, Ain Shams University, Cairo, Egypt \\ Corresponding author: Abeer El-Sayed Metwally Mohamed, Mobile: +201117630373, E-mail address: top_girls.2110@hotmail.com
}

\begin{abstract}
Objective: It was aimed at investigating the role of lung ultrasonography in assessment of pneumonia as compared to chest $\mathrm{x}$-ray in pediateric age group.

Purpose: to evaluate the ultrasound efficiency in the assessment of pneumonia in pediatric age group as compared to chest $\mathrm{x}$-ray.

Methodology: This study was carried out at the general pediatric wards and PICUs of El-Demrdash hospital, Ain Shams University.40 patients were clinically diagnosed with pneumonia. For every child included in the study, a chest $\mathrm{x}$-ray and a chest US were performed.

Result: the detection rate of consolidative patches was higher in ultrasound compared to the x-ray technique.

Conclusion: Lung ultrasound is safe and accurate for the diagnosis suspected cases of community acquired pneumonia and it is more sensitive than chest X-ray and allows a radiation free technique for detection of consolidations in children, thus reducing radiation exposure in this population.
\end{abstract}

Keywords: Lung Ultrasonography (LUS), Chest x-ray (CXR), Radiation, Imaging tool, Consolidation.

\section{INTRODUCTION}

Pneumonia is the single largest infectious cause of death in children worldwide. Pneumonia killed 920136 children under the age of 5 in 2015, accounting for $16 \%$ of all deaths of children under five years old ${ }^{(1)}$.

Diagnosis of community acquired pneumonia CAP) is done clinically but with poor diagnostic specificity ${ }^{(2,3)}$.

Chest X-ray (CXR) is considered as the first imaging step for further evaluation. Even though plain radiographs have small amounts of radiation dose exposure of about $0.01-1.5 \mathrm{mSv}$, children are more susceptible to nondeterministic stochastic effects of radiation than adults ${ }^{(4,5)}$.

In addition, the interpretation of CXR findings is dependent on the quality of the film and the expertise of the reader ${ }^{(6,7)}$.

The use of ultrasound for the evaluation of the lung is relatively recent. Lung ultrasound (LUS) is inexpensive, portable and non-ionizing imaging tool. It is relatively easy to teach ${ }^{(8)}$.

The various studies had shown that LUS performs well in adults ${ }^{(9,10)}$.

Lung ultrasound (LUS) is being increasingly used with children and neonates under various thoracic conditions $(11,12)$. It is therefore, and due to the accumulation of information and knowledges about radiation exposure hazards of using CXR with children, LUS technique was introduced and examined as a possible, useful, and safe tool instead of using CRX.

\section{AIM OF THE WORK}

The aim of this study was to evaluate the ultrasound efficiency in the assessment of pneumonia in pediatric age group compared to using chest $\mathrm{x}$-ray technique.

\section{PATIENT AND METHODS}

The study was performed on 40 Egyptian children (males and females), admitted during the period from June 2017 to June 2018 in the general pediatric wards and PICUs of El-Demrdash Hospital, Ain Shams University. The study was approved by the Ethics Board of Ain Shams University.

Children presenting with clinical picture of pneumonia were enrolled in the study. This symptoms included fever, cough, lethargy, chest pain and an acute onset of symptoms and signs of respiratory distress including tachypnea, retractions, grunting and cyanosis in addition to auscultatory findings associated with diminished air entry, fine rales and bronchial breath sounds.

Inclusion criteria: Clinical signs and symptoms of pneumonia (cough associated with abnormal lung auscultation or percussion and fever of unknown origin lasting longer than 5 days). Children aged from 0 to 16 years. Presence of clinical driven x-ray. Availability of a pediatric sonographer expert in LUS.

Exclusion Criteria: Unwilling parents/guardians. Contraindications with radiological exposure (chromosomal breaking syndromes, e.g. (ataxia Telangectasia, fragile $\mathrm{X}$ syndrome and Wiskott Aldrich syndrome). 
All included children were subjected to: History and clinical examination: Demographic data included age, gender. Symptoms of dyspnea, cough,expectoration \& fever. Full clinical examination including general examination fever, heart rate, respiratory rate. Imaging For every child included in the study, a chest $\mathrm{X}$-ray and a chest US were performed.

Chest $\mathrm{X}$ ray: Plain chest $\mathrm{X}$-ray was done in frontal view, either in the antro-posterior reclining or in the postro-anterior upright projection, depending on the age of the patient.

Lung ultrasonography: It was performed on GE logic P9 Ultrasound machine with superficial probe of 6-12 MHZ and convex probe of 1-5 MHZ was used in some cases, when this was considered necessary by the examiner, given the depth of the lesion. To cover the whole lung surface, the thorax was divided into three regions, anterior, posterior and lateral. Each region was scanned in the longitudinal and transvers plane, up-down and medial-lateral, respectively. The anterior and lateral regions of the chest were examined with the patient in supine position. The posterior region was examined in prone position or in a sitting position, facing away from the examiner. Known to the clinical findings. LUS was performed by trained paediatric sonologist blinded to CXR findings. The results of US imaging were compared to the interpretation of chest $\mathrm{X}$-rays.

Statistical analysis: Data were analyzed using Statistical Program for Social Science (SPSS) version 17 (SPSS Inc., Chicago, IL, USA). Quantitative data were expressed as range, mean \pm standard deviation (SD). Qualitative data were expressed as number and percentage.

\section{RESULTS}

A total of 40 patients were included in the study, 24 male $(60 \%)$ and 16 female $(40 \%)$. The mean patient age was $3.82 \pm 4.3$ years (range: 0.08 (1 month) -15 years).

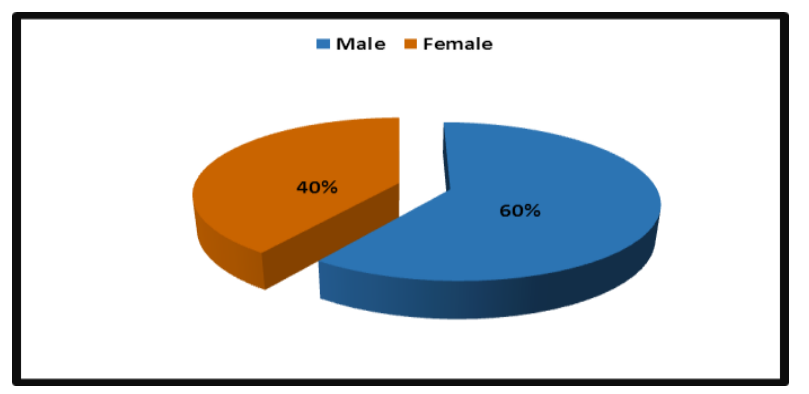

Figure (1): Pie chart demonstrating percentage of male and female patients.
Table (1): Demonstrating comparison between chest X-Ray and chest ultrasound regarding consolidation, air bronchogram and pleural effusion.

\begin{tabular}{|c|c|c|c|c|c|c|}
\hline & X-Ray & ultrasound & \multicolumn{2}{|c|}{$\begin{array}{c}\text { Wilcoxcon } \\
\text { Signed-Rank Test }\end{array}$} & \multirow{2}{*}{ significance } \\
\cline { 2 - 6 } & no. = 40 & no. = 40 & w & z value & p-value & \\
\hline Consolidation & $\begin{array}{c}18 \\
(45 \%)\end{array}$ & $28(70 \%)$ & 18 & -2.3541 & 0.01878 & S \\
\hline Air bronchogram & $9(23 \%)$ & $25(63 \%)$ & 21 & -3.1359 & 0.00168 & $\mathrm{~S}$ \\
\hline Pleural effusion & $9(23 \%)$ & $19(48 \%)$ & 0 & -2.8031 & 0.00512 & $\mathrm{~S}$ \\
\hline Pneumothorax & $1(3 \%)$ & $1(3 \%)$ & - & - & - & - \\
\hline Abscess & $2(5 \%)$ & $3(8 \%)$ & - & - & - & - \\
\hline
\end{tabular}

The previous table shows that there was statistically significant difference between chest XRay and chest ultrasound in detection of consolidation, air bronchogram and pleural effusion.

18 patients (45\%) were positive for consolidation by chest X-Ray, while 29 patients (73\%) were positive by chest Ultrasound.

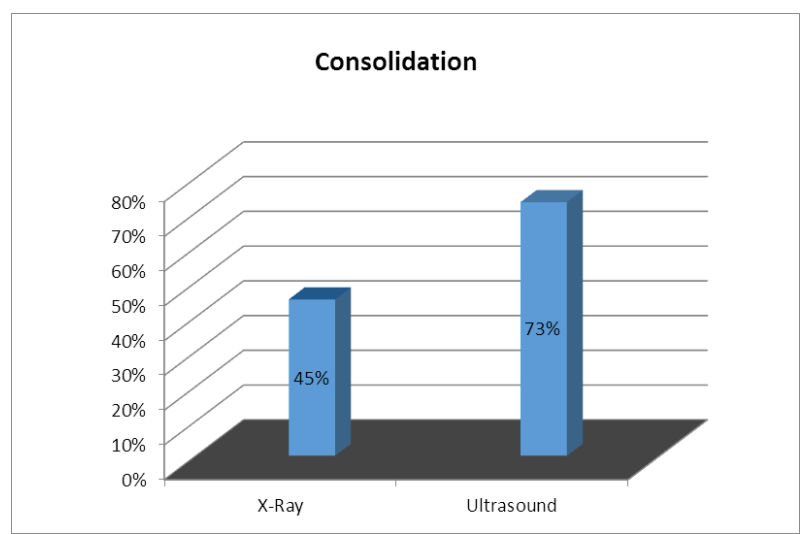

Figure (2): Bar chart demonstrating consolidation detected by chest X-ray and ultrasound.

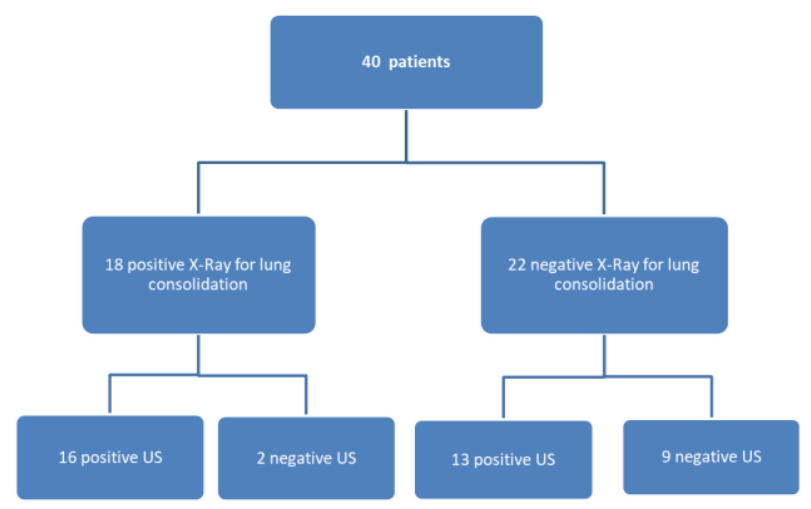

Figure (3): Confrontation of chest X-rays and US imaging: Positive versus negative cases for consolidartion.

Nine patients $(23 \%)$ were positive for air bronchogram in the consolidated areas by chest XRay, while 25 patients $(63 \%)$ were positive by chest Ultrasound. 


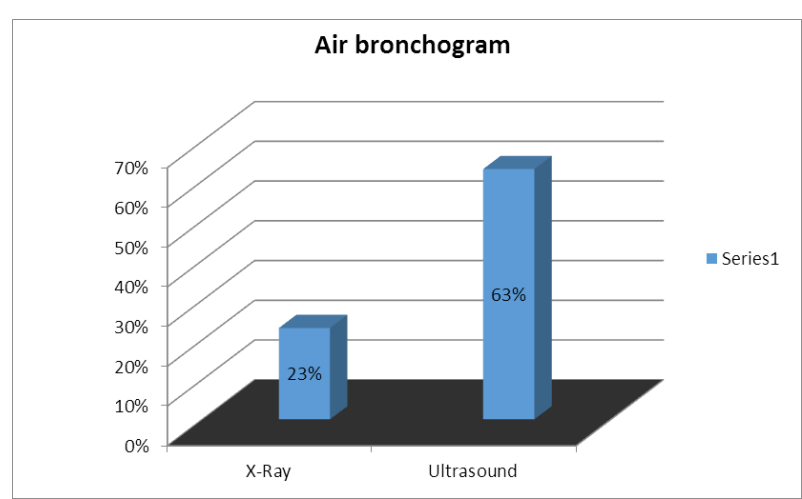

Figure (4): Bar chart demonstrating air bronchogram detected by chest X-ray and ultrasound.

Nine patients $(23 \%)$ were positive for pleural effusion by chest X-Ray, while 19 patients (48\%) were positive by chest Ultrasound.

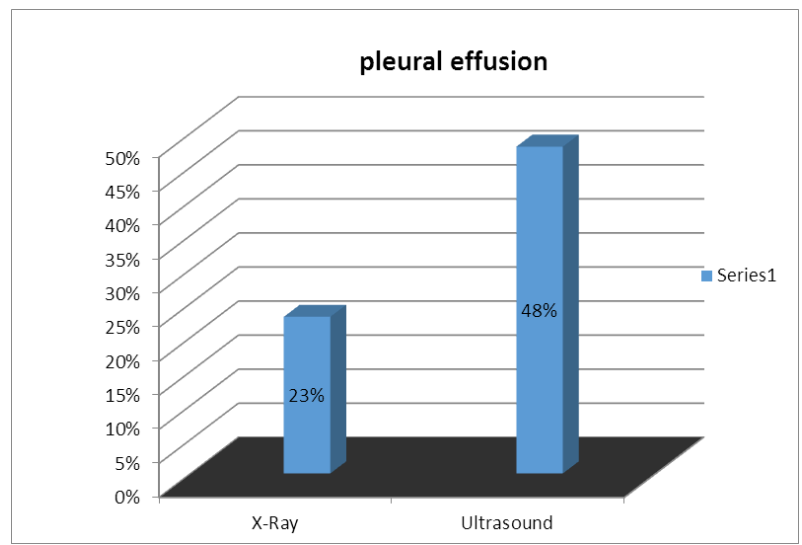

Figure (5): Bar chart demonstrating pleural effusion detected by chest X-ray and ultrasound.

Two patients (5\%) were positive for lung abscess by chest X-Ray, while 3 patients $(8 \%)$ were positive by chest Ultrasound.

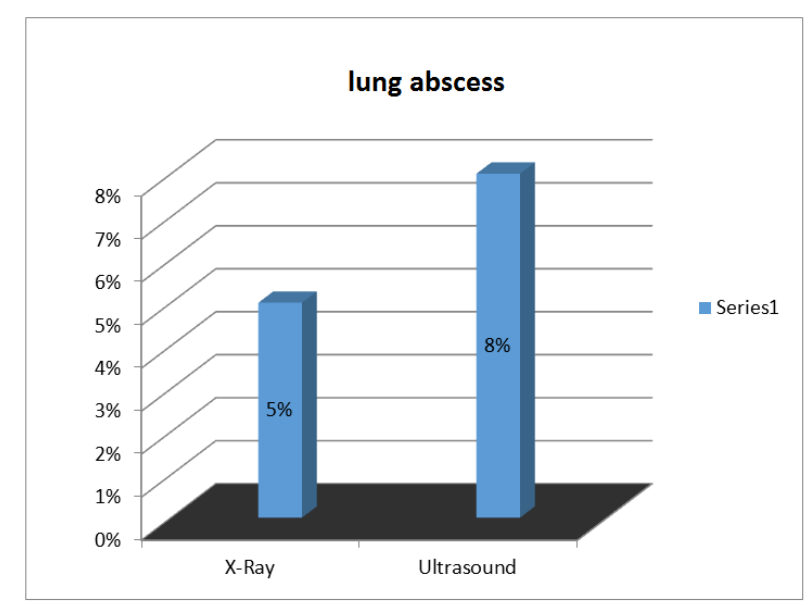

Figure (6): Bar chart demonstrating lung abscess detected by chest X-ray and ultrasound.
One patient (3\%) was positive for pneumothorax by chest X-Ray also 1 patient (3\%) was positive by chest Ultrasound.

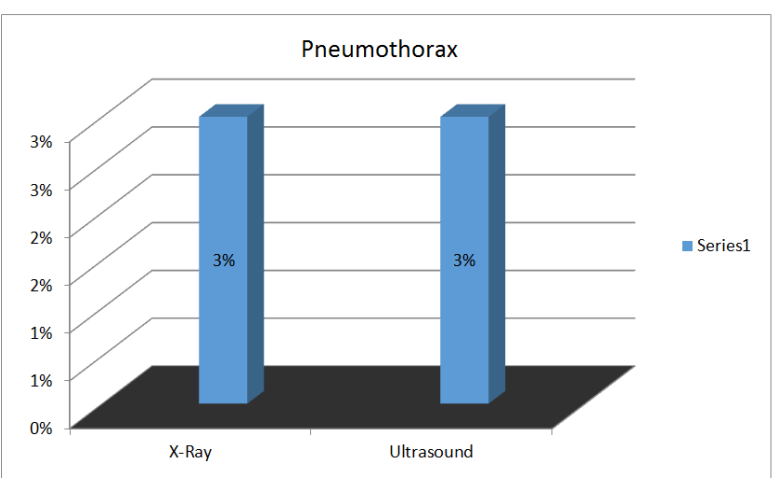

Figure (7): Bar chart demonstrating pneumothorax detected by chest X-ray and ultrasound.

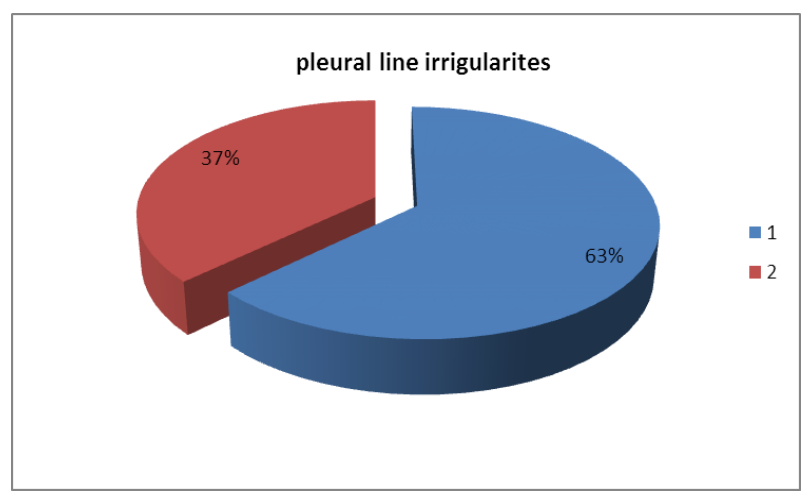

Figure (8): Pie chart demonstrating percentage of pleural line irregularities detected by chest ultrasound.

25 patients (63\%) show pleural line irregularities detected by chest ultrasound, while fluid bronchogram is seen in one patient (3\%).

Table (2): Demonstrating comparison between chest X-Ray and chest ultrasound regarding number of consolidation lesions.

\begin{tabular}{|l|c|c|c|c|}
\hline \multirow{2}{*}{$\begin{array}{c}\text { Number of } \\
\text { consolidation lesions }\end{array}$} & X-Ray & Ultrasound & \multicolumn{2}{|c|}{ Chi-square test } \\
\cline { 2 - 5 } & no. = 40 & no. =40 & x2 & P-value \\
\hline $\begin{array}{l}\text { Consolidation } \\
\text { confined to 1 lobe }\end{array}$ & $13(33 \%)$ & $16(40 \%)$ & & \\
\hline $\begin{array}{l}\text { Consolidation } \\
\text { in more than 1 lobe }\end{array}$ & $5(13 \%)$ & $19(48 \%)$ & & 0.06 \\
\hline
\end{tabular}

No statistically significant difference found between chest X-Ray and chest ultrasound in detection of consolidation in one lobe or more than one lobe. 


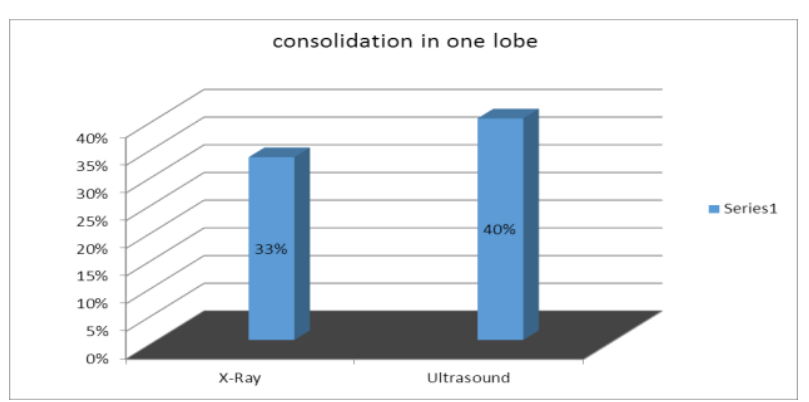

Figure (9): Bar chart demonstrating consolidation detected in one lobe by chest X-ray and ultrasound.

13 patients $(33 \%)$ show consolidation limited to one lobe by chest X-Ray, while 16 patients (40\%) show consolidation limited to one lobe by chest Ultrasound.

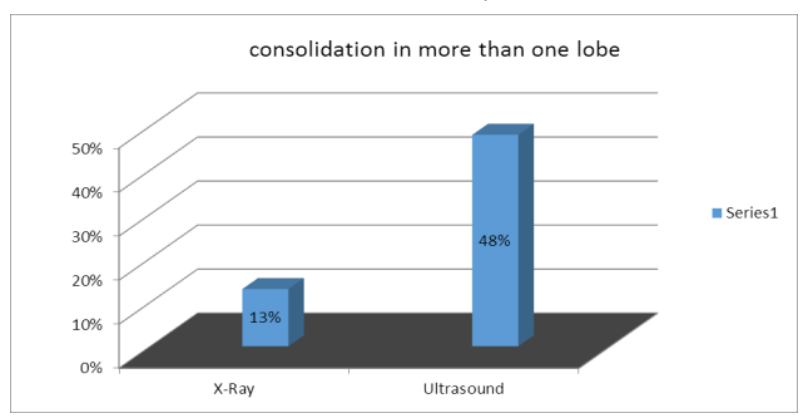

Figure (10): Bar chart demonstrating consolidation detected in one lobe by chest X-ray and ultrasound.

5 patients $(13 \%)$ show consolidation in more than one lobe by chest X-Ray, while 19 patients $(48 \%)$ show consolidation in more than one lobe by chest Ultrasound.

Detection rate of consolidation was $45 \%$ by chest $\mathrm{X}$-Ray, while detection rate by chest ultrasound was $72 \%$.

With contrary to other factors such as pleural effusion and pleural line abnormalities ultrasound was suggestive of pneumonia (90\%) while CXR was suggestive of pneumonia in (47\%).

16 patients were found to have CT chest in our PACS which were requested by the pediatrician.

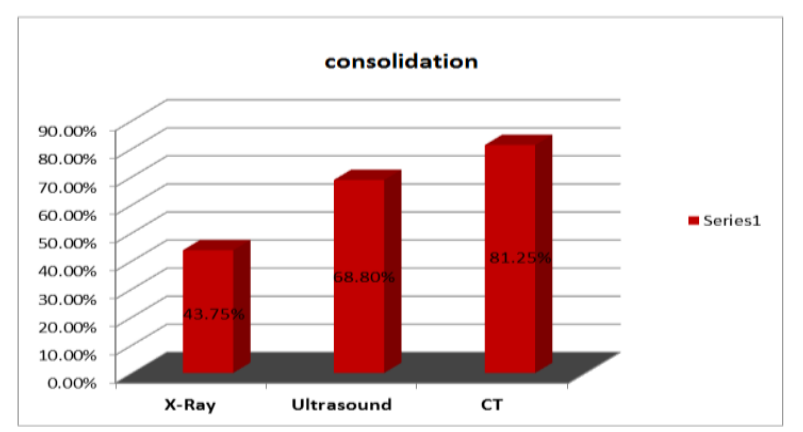

Figure (11): Bar chart demonstrating consolidation detected by chest X-ray, ultrasound and CT.
7 patients $(43.75 \%)$ were positive for consolidation by chest X-Ray, 11patients (68.8\%) were positive by chest Ultrasound, while 13 patient out of 16 patient $(81.25 \%)$ proved to have consolidation by CT. LUS was normal in 2 patients who were positive by CT while CXR failed to detect abnormality in 9 patients. Sensitivity of chest X-Ray in detection of consolidation $=68.42 \%$. Specificity of chest X-Ray in detection of consolidation $=100 \%$. Sensitivity of chest ultrasound in detection of consolidation $=86.67 \%$. Specificity of chest ultrasound in detection of consolidation $=100 \%$.

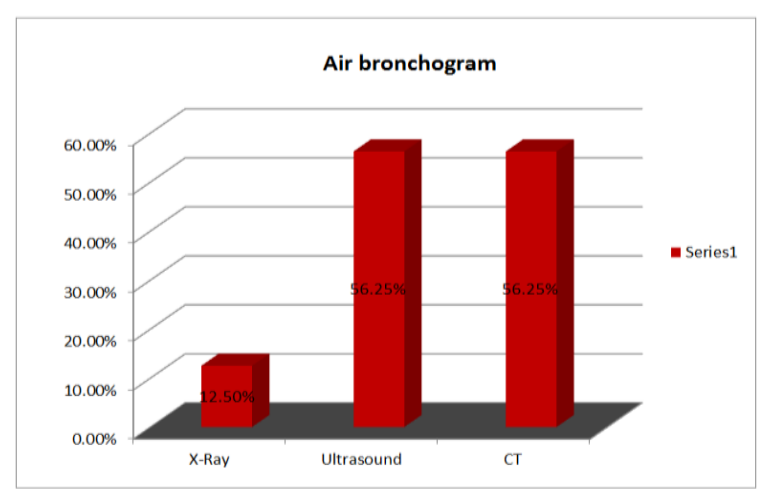

Figure (12): Bar chart demonstrating air bronchogram detected by chest X-ray, ultrasound and CT.

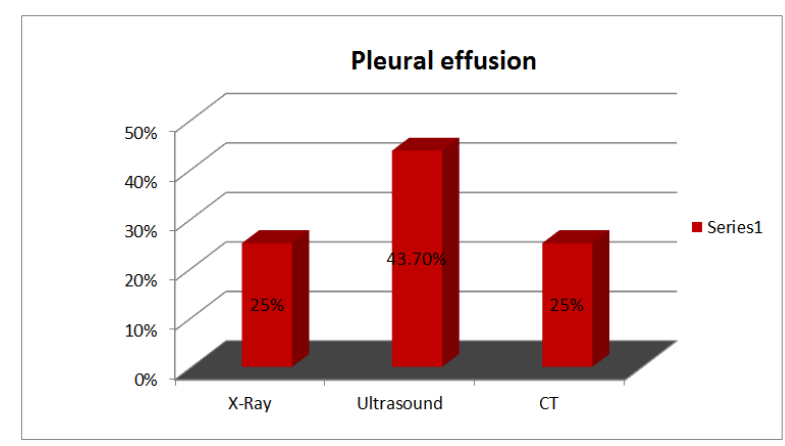

Figure (13): Bar chart demonstrating pleural effusion detected by chest X-ray, ultrasound and CT.

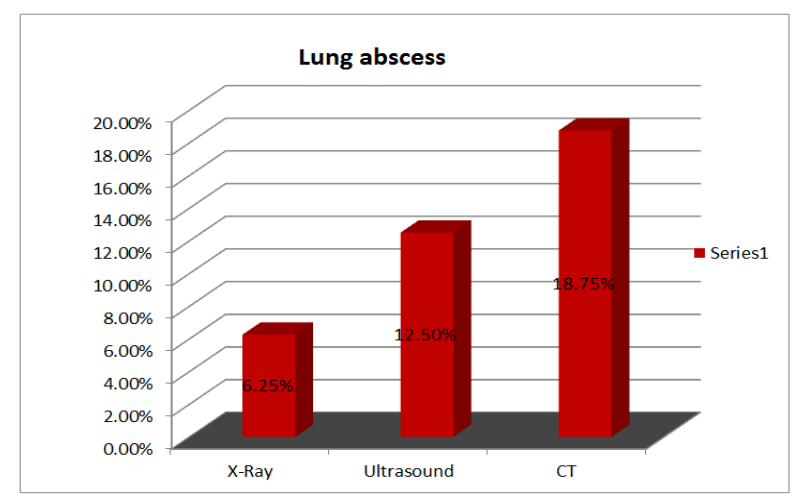

Figure (14): Bar chart demonstrating lung abscess detected by chest X-ray, ultrasound and CT. 


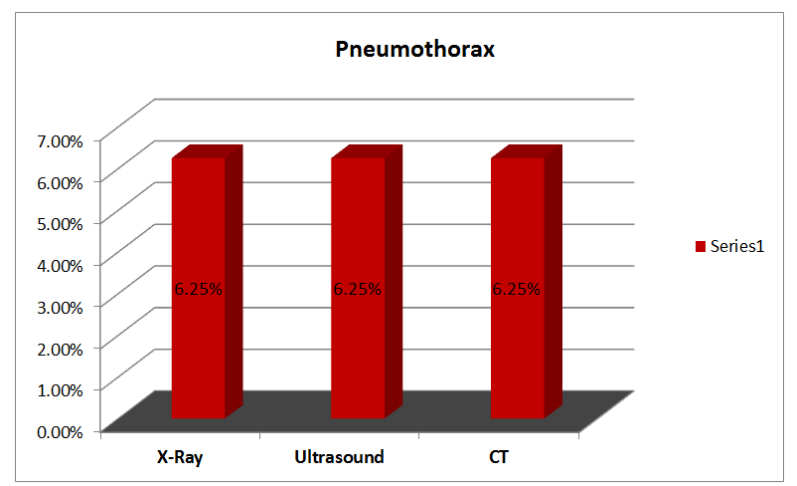

Figure (15): Bar chart demonstrating pneumothorax detected by chest X-ray, ultrasound and CT.

\section{DISCUSSION}

Early diagnosis and management of pneumonia are critical to short- and long-term health outcomes. Clinical examination is highly sensitive but lacks specificity and results in over diagnosis contributing to the overuse of antibiotics ${ }^{(13)}$.

CXR is considered the test of choice for further evaluation. The main limitations of radiography is the risk of damage from ionizing radiation with a greater risk than adults because children have more rapidly dividing cells and increased life expectancy ${ }^{(14)}$. Other concerns with radiography are great variability in the interpretation lack of reproducibility and delay in availability of the film. Also in complicated pneumonia CXR is less reliable and chest computed tomography (CT) scan is known to be the gold standard. However, its use has been discouraged due to high radiation, high cost and the need for sedation in young children ${ }^{(15)}$.

Medical radiation exposure is increasing rapidly. The radiological risk is cumulative in nature. The chest is the most frequently evaluated region of the body in children. There is research that suggests hepatoblastoma risk may increase due to repeated chest $\mathrm{X}$-rays in intensive care unit patients ${ }^{(14)}$. Unfortunately, pediatricians are often unaware of these risks. It is often possible to significantly decrease medical radiation exposure without compromising patient care.

Weinberg et al. ${ }^{(16)}$ first described the use of LUS in evaluating CAP. Subsequent studies have demonstrated that LUS is able to diagnose pneumonia in adults with high accuracy. Later on studies had demonstrated high efficacy of LUS in diagnosing pneumonia in children. Recently LUS has been indicated as a clinically useful diagnostic tool in pediatric patients with suspected pneumonia. The
LUS features of pneumonia mainly included sub pleural lung consolidation, pleural line abnormalities and synpneumonic pleural effusions.

In our study, the various LUS findings are close to the data in the literature. The detection of pneumonia using LUS was better $(90 \%)$ than with chest radiography $(47 \%)$. LUS was able to detect consolidation in $29(73 \%)$ patients as compared to $18(45 \%)$ patients by CXR of total 40 patients. LUS was normal in 11 patients while CXR failed to detect abnormality in 22 patients.

Ho et al. (17) also found that chest radiography able to detect 151 (92.6\%), whereas LUS detected 159 (97.5\%) out of 163 patients with pneumonia.

Tirdia et al. ${ }^{(18)}$ found that the detection of pneumonia using LUS was better $(97.84 \%)$ than with chest radiography $(90.64 \%)$. LUS was able to detect consolidation in $130(93.53 \%)$ patients as compared to $107(76.97 \%)$ patients by CXR of total 139 patients, the difference between these two studies and our study could be attributed to different sample size.

In our study LUS had a sensitivity of $86.67 \%$ and specificity of $100 \%$, compared to sensitivity of $68.42 \%$ and specificity of $100 \%$ by CXR, Pereda in meta-analysis found that LUS had a sensitivity of $96 \%$ and specificity of $93 \%$. Other published data also showed that LUS is more sensitive than CXR in the diagnosis of pneumonia in children ${ }^{(19)}$.

From 40 patients included in this study there were 18 positive cases for consolidation by chest $\mathrm{X}$ ray, two of them were negative by ultrasound, one of the 2 cases the consolidation was located in the perihilar region and other in the right upper lung zone. One possible explanation to these errors in detection by US may be suggested. These areas of consolidation may lack contact with pleural surface and thus invisible on US. Despite this technical limitation, according to Lichtenstein et al., $98 \%$ of pneumonias in adults have a pleural contact taking into consideration that a child's pulmonary volume is smaller. The rate of pleural contact in pediatric pneumonia should be at least equal to that in adults ${ }^{(17)}$.

Areas of lung where errors in detection by US could have been expected, such as the retroscapular region or the lung apex finally didn't represent a problem. These areas were accessible to US, though the thoroughness of examination and obligation to cover the whole lung when scanning for pneumonia must be stressed. 
In the other 22 patients with negative CXR 13 of them had positive ultrasound findings and their clinical course was consistent with pneumonia.

Also in our study 9 patients $(23 \%)$ were positive for air bronchogram in the consolidated areas by chest X-Ray, while 25 patients (63\%) were positive by chest Ultrasound, and according to the study done by Hajalioghli et al. they declared that the presence of air bronchogram is an important finding that significantly increased the diagnostic accuracy of US in their work as sonographic air bronchogram were indicative of pneumonia in contrary to atelectasis. ${ }^{(20)}$

There are methodological limitations to our study. Chest X-ray is not a perfect gold standard. It requires strict technical criteria, especially in children, and inter-observer variability in interpretation is well known. Incorrect positioning and insufficient lung expansion on inspiration have a great influence on the quality of the X-ray. On the other hand, certain areas of the lung, such as the bases, superposed to the diaphragm, are more difficult to interpret. In this study chest X-ray was not performed systematically. International guidelines are variable on this point: the American Society of Infectious Diseases guidelines still indicate front and lateral chest X-ray for all children suspected of pneumonia, while those British Thoracic Society specifically mention that lateral view is not necessary. In our pediatric radiology department,in order to limit radiation exposure as much as possible, a lateral chest X-ray is never done routinely, but only when the interpreting radiologist consider that this is necessary. In the population included in the study only three cases had lateral chest X-ray ${ }^{(21)}$.

As with other ultrasound applications, LUS can be operator dependent. the correct diagnosis cannot be made if the operator is insufficiently trained or inexperienced. Fortunately the learning curve for LUS appears to be easier and faster than other ultrasound applications ${ }^{(22)}$.

Other limitation to this study. The sample size was small, and therefore, our results need to be validated by further studies.

Thus, our study demonstrates that LUS is safe and accurate for the diagnosis suspected cases of CAP and it is more sensitive than CXR and allows a radiation free technique for detection of consolidations in children, thus reducing radiation exposure in this population.
Some technical advantages such as shorter thoracic width, thinner chest wall, and small lung mass theoretically enable LUS examination in children easier than in adults. 19

The short duration of lung ultrasound imaging supports the assumption that US can be performed in everyday practice, depending on the setup of each radiology department.

\section{REFERENCES}

1. World Health Organization Pneumonia Fact sheet (2016): www.who.int/mediacentre/ factsheets/fs331.

2. Harris M, Clark S and Coote $N$ (2011): British Thoracic Society guidelines for the management of community acquired pneumonia in children. Thorax, 66(2): ii1-23.

3. Shah S, Bachur R, Kim D et al. (2010): Lack of predictive value of tachypnea in the diagnosis of pneumonia in children. Pediatric Infectious Disease Journal, 29(5): 406-9.

4. Park MY and Jung SE (2016): Patient Dose Management: Focus on Practical Actions. Journal of Korean Medical Science, 31(1): S45-54.

5. Gargani $L$ and Picano $\mathbf{E}$ (2015): The risk of cumulative radiation exposure in chest imaging and the advantage of bedside ultrasound. Critical Ultrasound Journal, 7: 4.

6. Williams GJ, Macaskill $P$ and Kerr $M$ (2013): Variability and accuracy in interpretation of consolidation on chest radiography for diagnosing pneumonia in children under 5 years of age. Pediatr Pulmonol., 48(12): 1195-200.

7. Wingerter SL, Bachur RG, Monuteaux MC et al. (2012): Application of the world health organization criteria to predict radiographic pneumonia in a USbased pediatric emergency department. Pediatr Infect Dis J., 31(6): 561-4.

8. Solomon SD and Saldana F (2014): Point-ofcare ultrasound in medical educationstop listening and look. N Engl J Med., 370(12): 1083-5.

9. Chavez MA, Shams $\mathbf{N}$ and Ellington LE (2014): Lung ultrasound for the diagnosis of pneumonia in adults: a systematic review and meta-analysis. Respir Res., 15: 50. 
10. Ye X, Xiao H, Chen B et al. (2015): Accuracy of Lung Ultrasonography versus Chest Radiography for the Diagnosis of Adult CommunityAcquired Pneumonia: Review of the Literature and MetaAnalysis. PLoS One, 10(6): e0130066.

11. Chen SW, Zhang MY and Liu J (2015): Application of lung ultrasonography in the diagnosis of childhood lung diseases. Chinese Medical Journal, 128(19): 2672.

12. Cattarossi L (2013): Lung ultrasound: its role in neonatology and pediatrics. Early Hum Dev., 89(1): S17-9.

13. Zimmerman DR, Kovalski N, Fields $\mathrm{S}$ et al. (2012): Diagnosis of childhood pneumonia: clinical assessment without radiological confirmation may lead to over treatment. Pediatric Emergency Care, 28(7): 646-9.

14. Park MY and Jung SE (2016): Patient Dose Management: Focus on Practical Actions. Journal of Korean Medical Science, 31(1): S45-54.

15. Gargani $L$ and Picano $E$ (2015): The risk of cumulative radiation exposure in chest imaging and the advantage of bedside ultrasound. Critical Ultrasound Journal, 7: 4.

16. Weinberg B, Diakoumakis EE, Kass EG et al. (1986): The air bronchogram: sonographic demonstration. American Journal of Roentgenology, 147: 593-5.

17. Ho MC, Ker CR, Hsu JH et al. (2015): Usefulness of lung ultrasound in the diagnosis of community-acquired pneumonia in children. Pediatrics and Neonatology, 56(1): 40-5.

18. Tirdia PR, Vajpayee S, Singh J et al. (2016): Accuracy of lung ultrasonography in diagnosis of community acquired pneumonia in hospitalized children as compared to chest $\mathrm{x}$ ray. International Journal of Contemporary Pediatrics, 3(3): 1026-31.

19. Ianniello S, Piccolo CL, Buquicchio GL et al. (2016): First-line diagnosis of pediatric pneumonia in emergency: lung ultrasound (LUS) in addition to chest-X-ray and its role in followup. The British Journal of Radiology, 89: 1061.

20. Hajalioghli P, Nemati M, Dinparast Saleh L et al. (2016): Can chest computed tomography be replaced by lung ultrasonography with or without plain chest radiography in pediatric pneumonia? Journal of Thoracic Imaging, 31(4): 247-252.
21. Bradley JS, Byington CL, Shah SS et al. (2011): The management of communityacquired pneumonia in infants and children older than 3 months of age: clinical practice guidelines by the Pediatric Infectious Diseases Society and the Infectious Diseases Society of America. Clinical Infectious Diseases, 53(7): $25-76$

22. Bedetti G, Gargani L, Corbisiero A et al. (2006): Evaluation of ultrasound lung comets by hand-held echocardiography. Cardiovascular Ultrasound, 4(1): 34. 